\title{
MRI Findings in Post-Operative Bilateral Posterior Ischemic Optic Neuropathy
}

\author{
Nirali P. Bhatt ${ }^{1 *}$, Robert E. Morales ${ }^{2}$, Michaela K. Mathews ${ }^{1}$ \\ ${ }^{1}$ Department of Ophthalmology and Visual Sciences, University of Maryland School of Medicine, Baltimore, USA; ${ }^{2}$ Department of \\ Diagnostic Radiology and Nuclear Medicine, University of Maryland School of Medicine, Baltimore, USA. \\ Email: ${ }^{*}$ niralipbhatt@gmail.com
}

Received April 19 ${ }^{\text {th }}, 2013$; revised May 20 ${ }^{\text {th }}, 2013$; accepted June $15^{\text {th }}, 2013$

Copyright @ 2013 Nirali P. Bhatt et al. This is an open access article distributed under the Creative Commons Attribution License, which permits unrestricted use, distribution, and reproduction in any medium, provided the original work is properly cited.

\begin{abstract}
A 62-year-old female complained of vision loss following multiple abdominal surgeries for mesenteric ischemia. The patient's visual acuity was no light perception (NLP) in the right eye and hand motion (HM) at 1' in the left eye. Both pupils were unreactive and no relative afferent pupillary defect was noted. Anterior segment and fundus examination were unremarkable. T1 and T2 weighted MRI imaging of the brain was normal but diffusion weighted imaging (DWI) revealed areas of bright signal within both intraorbital optic nerves, confirming the diagnosis of posterior ischemic optic neuropathy.
\end{abstract}

Keywords: Optic Nerve; Post-Operative Posterior Ischemic Optic Neuropathy; MRI; DWI

\section{Case Report}

A 62-year-old Hispanic female with a previous history of diabetes, hypertension, and glaucoma underwent repeated laparotomies and bowel resections for mesenteric ischemia. During the procedures, several hypotensive episodes were recorded. After the last surgery, the patient complained of having difficulty seeing objects in front of her. The patient's visual acuity was found to be reduced to no light perception (NLP) in the right eye and hand motion (HM) at 1' in the left eye. Both pupils were unreactive to light. No relative afferent pupillary defect was present. The patient had normal ocular motility and alignment. Confrontational visual fields could not be obtained reliably. Anterior segment examination showed moderate cataracts in both eyes. Both optic nerves showed a cup-to-disc ratio of 0.4 , without pallor or edema. The retina, macula, periphery and retinal vessels were unremarkable. Histopathology of the surgical specimen as well as laboratory studies revealed no evidence of vasculitis. The patient underwent MRI and magnetic resonance angiography (MRA) of the brain. No acute infarct was identified within the brain parenchyma with findings suggestive of chronic small vessel ischemic change in the white matter (Figure 1). No evidence of advanced edema within the optic nerves was identified

${ }^{*}$ Corresponding author.
(Figure 2). MRA of the brain showed no major branch vascular occlusion. DWI revealed increased signal within the right greater than left optic nerves (Figure 3). These areas demonstrated corresponding decreased signal on

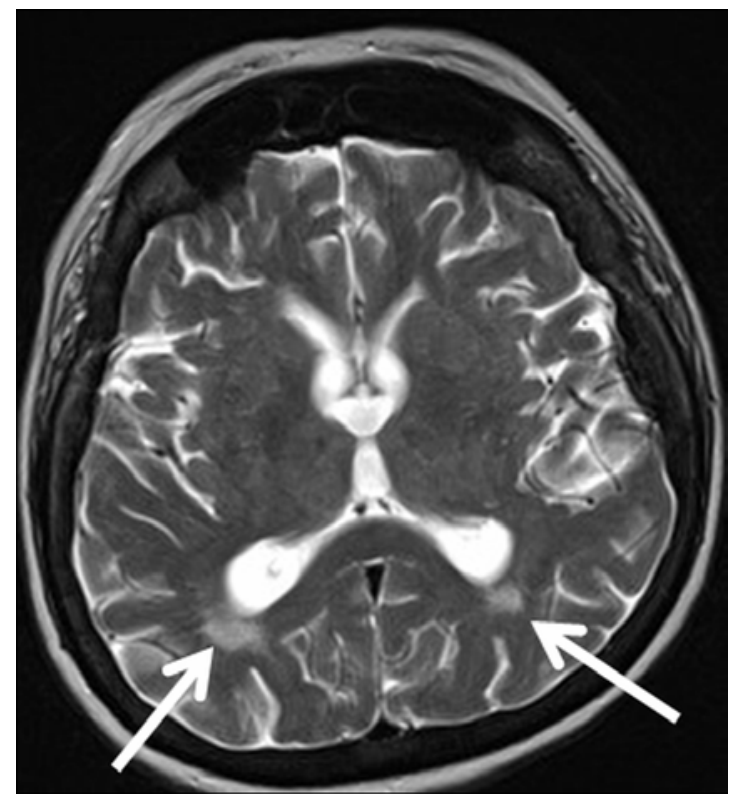

Figure 1. T2-weighted image of the brain shows nonspecific increased signal within the periventricular white matter suggestive of chronic small vessel ischemic change (arrows). 


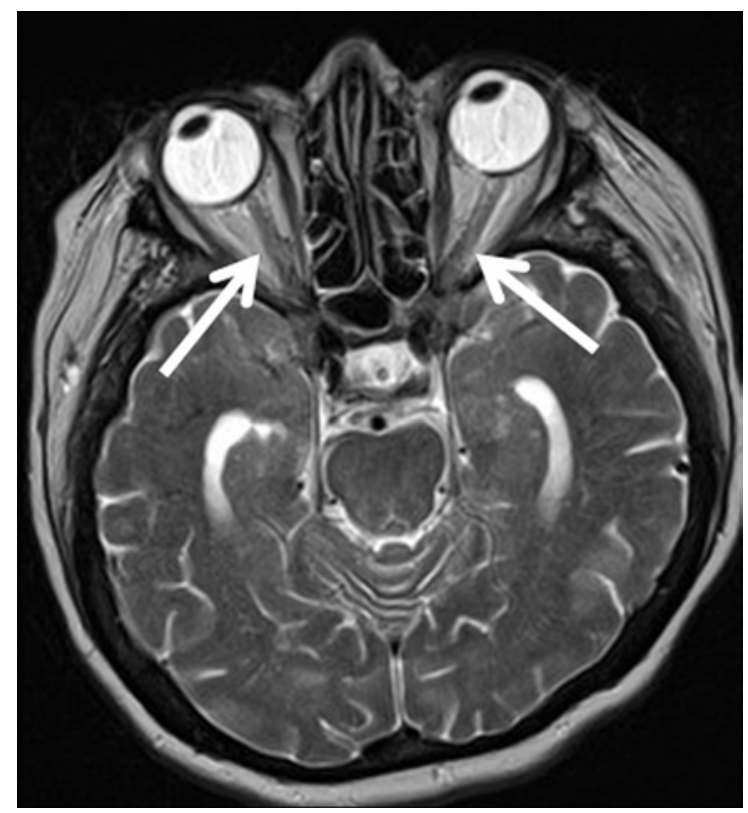

Figure 2. T2-weighted image at the level of the orbits shows normal signal within the optic nerves bilaterally (arrows).

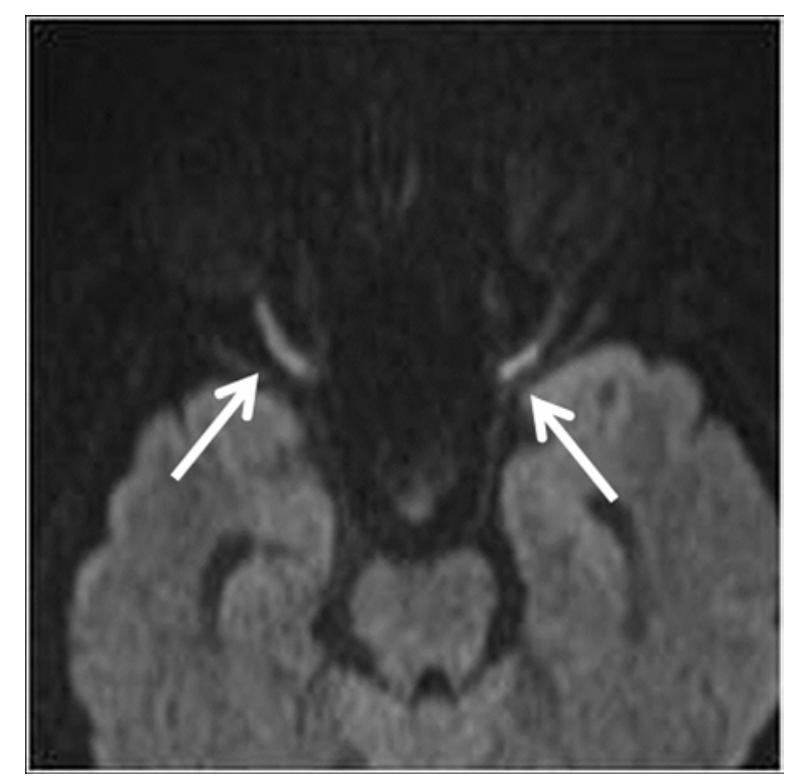

Figure 3. DWI shows increased signal within the right greater than left intraorbital optic nerves (arrows).

the apparent diffusion coefficient (ADC) map, consistent with true restricted diffusion (Figure 4). This finding is consistent with bilateral ischemic injury/infarction of the posterior portion of the optic nerves.

\section{Discussion}

Post-operative posterior ischemic optic neuropathy (PION) may be a difficult clinical diagnosis, especially when bilateral. Patient complaints of decreased visual acuity following surgery are often interpreted as "drowsi-

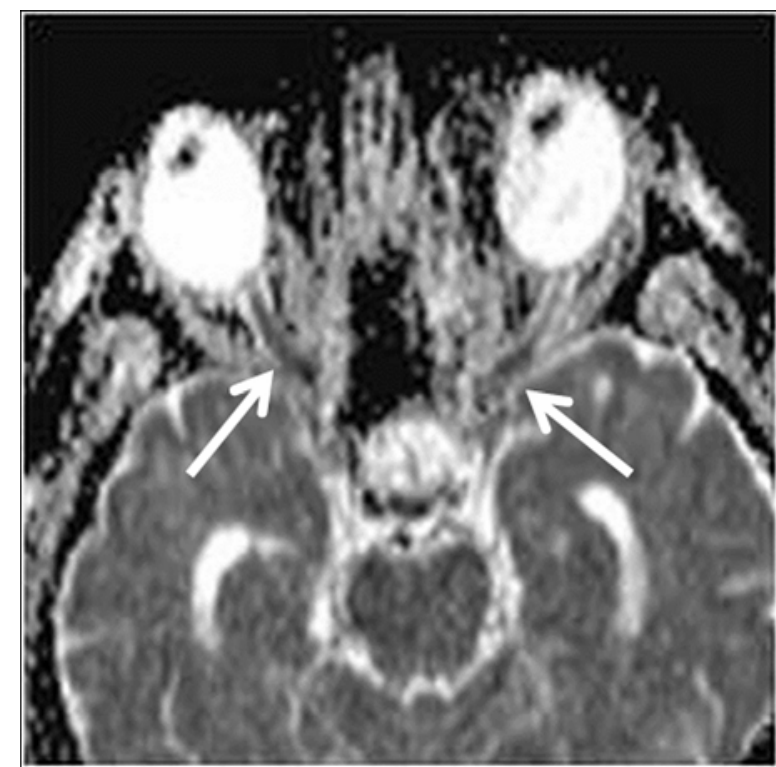

Figure 4. ADC map shows corresponding decreased signal confirming true restricted diffusion (arrows).

ness” after general anesthesia. If both optic nerves are affected, an APD is often absent. By definition, PION lacks optic disc edema, flame hemorrhages, and cotton wool spots that are diagnostic of anterior ischemic optic neuropathy (AION). The optic nerve looks healthy until optic nerve pallor develops up to 6 - 12 weeks after the initial event [1].

In most of these cases, intra-operative and/or post-operative anemia and hypovolemic hypotension are believed to be major risk factors [2-4]. The posterior segment of the optic nerve is relatively poorly vascularized, compared to the anterior portion, which is supplied by a vascular plexus derived from collateral branches of the ophthalmic artery and the central retinal artery. Near the lamina cribrosa, a rich capillary network arises from the circle of Zinn-Haller. The posterior segment of the optic nerve, in contrast, is supplied only by the pial capillary plexus that surrounds it. Only a small number of capillaries penetrate the nerve and extend to its central portion. As a result, the center of the posterior portion of the optic nerve is particularly susceptible to ischemia [5].

In acute ischemic injury, the development of cytotoxic edema results in a shift of water molecules from the extracellular to the intracellular space, with restricted diffusion across the cell membrane. This is demonstrated as increased signal on DWI rapidly after the insult. In contrast to infarctions within the brain parenchyma, where DWI can readily locate areas of ischemic damage, MRI changes in the optic nerve have only been described in a few cases of PION [6,7]. In our patient, DWI helped confirm the clinical diagnosis, by showing evidence of bilateral optic nerve ischemic injury/infarction. 


\section{REFERENCES}

[1] S. Dunker, H. Y. Hsu, J. Sebag and A. A. Sadun, "Perioperative Risk Factors for Posterior Ischemic Optic Neuropathy," Journal of the American College of Surgeons, Vol. 194, No. 6, 2002, pp. 705-710.

doi:10.1016/S1072-7515(02)01210-3

[2] S. S. Hayreh, "Posterior Ischaemic Optic Neuropathy: Clinical Features, Pathogenesis, and Management,” Eye, Vol. 18, 2004, pp. 1188-1206.

doi:10.1038/sj.eye.6701562

[3] L. A. Lee, N. J. Newman, T. A. Wagner, J. R. Dettori and N. J. Dettori, "Postoperative Ischemic Optic Neuropathy," Spine, Vol. 35, No. 9, 2010, pp. S105-S116.

[4] N. J. Newman, "Perioperative Visual Loss after Nonocular Surgeries,” American Journal of Ophthalmology, Vol. 145, No. 4, 2008, pp. 604-610. doi:10.1016/j.ajo.2007.09.016

[5] V. Purvin and B. Kuzma, "Intraorbital Optic Nerve Signal Hyperintensity on Magnetic Resonance Imaging Sequences in Perioperative Hypotensive Ischemic Optic Neuropathy,” Journal of Neuro-Ophthalmology, Vol. 25, No. 3, 2005, pp. 202-204.

doi:10.1097/01.wno.0000177295.52468.5b

[6] S. R. Sadda, M. Nee, N. R. Miller, V. Biousse, N. J. Newman and A. Kouzis, "Clinical Spectrum of Posterior Ischemic Optic Neuropathy,” American Journal of Ophthalmology, Vol. 132, No. 5, 2001, pp. 743-750. doi:10.1016/S0002-9394(01)01199-0

[7] M. S. Vaphiades, "Optic Nerve Enhancement in Hypotensive Ischemic Optic Neuropathy,” Journal of Neuro-Ophthalmology, Vol. 24, No. 3, 2004, pp. 235-236. doi:10.1097/00041327-200409000-00011 